\title{
Recuperación de la Morfología y Fisiología Maxilo Mandibular: Biomateriales en Regeneración Ósea
}

\author{
Maxillomandibular Morphology and Phisiology Recovery: Biomaterials in Bone Regeneration \\ *,**Gonzalo Oporto Venegas; *Ramón Fuentes Fernández; **Héctor Álvarez Cantoni \& *Eduardo Borie Echeverría
}

OPORTO, V. G.; FUENTES, F. R.; ÁLVAREZ, C. H. \& BORIE, E. E. Recuperación de la morfología y fisiología maxilomandibular: Biomateriales en regeneración ósea. Int. J. Morphol., 26(4): 853-859, 2008.

RESUMEN: Las caries dentales y la enfermedad periodontal son un problema de salud pública. En Chile, el $100 \%$ de la población de NSE medio - bajo y bajo presenta caries, con índices más altos en la IX Región. El 92.19\% de las personas entre 35 y 74 años presentan periodontitis. Ambas enfermedades pueden llevar a que el paciente pierda sus dientes. Existen técnicas que mejoran el pronóstico de tratamientos protésicos, tales como los implantes de titanio, que pueden requerir aumentar la cantidad de hueso para reestablecer la forma y función. Se muestra una revisión de la literatura sobre biomateriales en regeneración ósea. El biomaterial con mejores propiedades es el hueso autólogo (AI), sin embargo, existen alternativas, aloinjerto (AL) y matrices óseas. Los AI poseen mejor pronóstico, los de origen endocondral (EC) e intramembranoso (IM) con un 357\% y un 642\% más formación ósea que un AL de tipo IM (6). Los AI IM son más exitosos que los EC (IM 166\% más neoformación ósea). La disponibilidad de AI es limitada, por lo que es necesario utilizar biomateriales. AL mezclado con matriz ósea produce un $224 \%$ más hueso que AL IM solo. La utilización de membranas mejora la estabilidad a largo plazo de hueso IM y EC. AI EC son los biomateriales más exitosos. Dada su baja disponibilidad, pueden ser usados en conjunto con matrices. Las membranas han mostrado aumentar la estabilidad de los biomateriales.

PALABRAS CLAVE: Regeneración ósea; Materiales biocompatibles; Implantes dentales.

\section{INTRODUCCIÓN}

Las enfermedades de la cavidad oral constituyen un problema de salud pública para muchos países del mundo, en especial para aquellos en vías desarrollo o subdesarrollados. Esta afirmación encuentra bases en el componente socio cultural como factor etiológico, que poseen las personas que padecen las citadas patologías (huéspedes de la enfermedad), así como en el alto costo de los tratamientos odontológicos, lo que en ocasiones provoca que quienes requieren estas intervenciones se encuentren incapacitadas de pagar y acceder a tratamientos, sean éstos preventivos o curativos.

Es conocido que dentro de las patologías más prevalentes de la cavidad oral se encuentran las caries dentales y la enfermedad periodontal. En Chile, la realidad a este respecto es bastante compleja. En 1995 se estableció que en la Región Metropolitana, el 100\% de la población de niveles socioeconómicos medio - bajo y bajo, presentaba caries dentales, con índices COPD (dientes cariados, obturados y perdidos) superiores a 25 en personas mayores de 25 años de edad (Urbina et al. 1996). Posteriormente, en
1999, se establece que el 99\% de las mujeres embarazadas presentaba caries (Villagrán, 1999). El Ministerio de Salud de Chile (MINSAL) en el año 2003 (MINSAL, 2004) estableció que el 66\% de la población nacional se encontraba afectada por caries, con índices más altos en la Región de la Araucanía, donde la población de 12 años se encuentra afectada en un $97,8 \%$ por esta enfermedad, convirtiéndose en la región con el índice más alto a nivel nacional (MINSAL).

En cuanto a la enfermedad periodontal en Chile, la realidad no es mucho más alentadora. Se estableció en el año 2003, que el $92.19 \%$ de las personas entre 35 y 74 años presentan periodontitis, con un $90.89 \%$ de individuos afectados entre 35 y 44 años de edad, cifra que aumentó en el grupo etáreo comprendido entre 65 y 74 años, donde el $100 \%$ de las personas se encontraba afectada por esta patología (Gamonal et al. 1998).

Lo señalado cobra importancia ante el hecho de que ambas enfermedades, de no ser tratadas oportunamente, pueden llevar a que el paciente pierda sus piezas dentales, por

\footnotetext{
* Departamento de Odontología Integral, Facultad de Medicina, Universidad de La Frontera. Temuco, Chile.

** Carrera Principal de Especialización en Rehabilitación Protética de Alta Complejidad con Orientación en Prótesis Implanto-asistida y Prótesis Parcial Fija, Facultad de Odontología. Universidad de Buenos Aires, Buenos Aires, Argentina.
} 
lo que existiría una correlación efectiva entre la prevalencia de estas patologías y la pérdida de dientes. El año 2003, el MINSAL estableció que más del $70 \%$ de la población nacional manifiesta tener su dentadura incompleta.

Si a lo anterior se agrega el aumento de la esperanza de vida en Chile, es posible prever poblaciones envejecidas en el futuro, donde este problema será aun más importante, y la demanda de soluciones será mayor.

Las piezas dentales juegan un rol fundamental en el desarrollo de las actividades cotidianas del ser humano, sean biológicas como alimentación, así como en las relaciones sociales, donde la imagen influye tanto en la sociabilización cotidiana como en la consecución de un trabajo.

La carencia de piezas dentales afecta al ser humano desde la perspectiva física general, social y psicológica, especialmente en el ámbito de la autoestima, por lo tanto, poder rehabilitar la cavidad oral de pacientes desdentados cobraría real importancia desde las perspectivas biológica, social y psicológica.

En pacientes adultos desdentados parcial o totalmente, la base ha sido la rehabilitación oral por medio de prótesis. Sin embargo, existe una limitante; el éxito relativo de un tratamiento de rehabilitación oral va a depender, entre otros y muy a grosso modo, de la presencia de piezas dentarias para soportar y sostener una prótesis parcial removible o prótesis fija tradicional en pacientes parcialmente desdentados, o bien en el remanente óseo que cumpla estas funciones en pacientes desdentados totales que opten por prótesis completas removibles.

Existen técnicas que pueden mejorar el pronóstico de tratamientos protésicos, una de ellas es la colocación de implantes de titanio, técnica probada a nivel mundial con excelentes resultados (Olate et al. 2007). Sin embargo, esta solución también se hace compleja cuando los pacientes que requieren prótesis implanto asistidas presentan pobre remanente óseo (Olate et al.; Anitua 2000). Ante esta situación, la solución sería recuperar la cantidad de hueso perdido para reestablecer la forma y función del reborde alveolar, así como para crear el lecho que pueda recibir los implantes. Para esto se requiere realizar maniobras conducentes a lograr un aumento del volumen óseo existente, es decir, inducir regeneración ósea.

La presente revisión busca conocer los tipos de biomateriales y su tasa de éxito en la regeneración de la forma y función de rebordes alveolares para rehabilitación oral con prótesis implanto-asistida.
Regeneración ósea. La regeneración ósea es posible de obtener mediante la combinación de tres elementos: células viables, matriz extracelular y sustancias reguladoras insolubles (factores de crecimiento); sin dejar de mencionar factores locales que también son influyentes, como los entorno mecánico y vascular. La combinación de estos elementos generará un ambiente apropiado para la regeneración (Olate et al.; Anitua; Fuentes et al., 2005).

El éxito de la regeneración ósea se relaciona con tres mecanismos.

Osteogénesis: Proceso de formación y desarrollo de hueso nuevo. Los materiales osteogénicos se derivan o se encuentran formados por tejido implicado en crecimiento y reparación, por ejemplo, el hueso autólogo, es decir, del mismo paciente (Olate et al.; Anitua; Fuentes et al.).

Osteoinducción: Proceso de estimulación de la osteogénesis. Los materiales osteoinductivos son aquellos que se utilizan para mejorar la regeneración ósea, y el hueso puede crecer o extenderse por una zona donde normalmente no se encuentra. La regeneración ósea será estimulada por liberación de proteínas que facilitan la diferenciación celular. Son ejemplo de materiales osteoinductivos: a) el hueso autólogo en la fase de reabsorción que libera proteínas morfogenéticas; b) plasma rico en factores de crecimiento (PRGF) que estimulan la quimiotaxis, diferenciación y proliferación celular y c) las proteínas morfogenéticas (Olate et al.; Anitua; Fuentes et al.).

Osteoconducción: Proporciona la matriz o estructura física apropiada para la deposición de hueso nuevo. Los materiales osteoconductores son guías para el crecimiento óseo y permiten que se deposite el hueso nuevo, el cual se formará por sustitución progresiva. Son ejemplos de materiales osteoconductores: hueso autólogo, fibrina autóloga, hidroxiapatita reabsorvible, sulfato de calcio, fosfato tricálcico, fibrina liofilizada, hueso desmineralizado, cristales cerámicos bioactivos (Olate et al.; Anitua; Fuentes et al.) y otros.

Dentro de los procesos regenerativos existen además dos técnicas que vale la pena mencionar.

Regeneración tisular guiada (osteopromoción): Es la capacidad de inducir la formación ósea mediante la utilización de barreras, cuya finalidad es, por medio de una barrera física, que la angiogénesis por defecto provenga a partir del lecho receptor, y evitar la llegada de vasos a partir del conectivo adyacente. Estas barreras pueden ser reabsorbibles o no reabsorbibles (Olate et al.; Anitua; Fuentes et al.). 
Osteodistracción: Se basa en provocar una fractura y separar los fragmentos con instrumentos denominados osteodistractores, los que estirarán el coágulo de fibrina y se promoverá la formación de un puente óseo entre los fragmentos (Olate et al.; Anitua.).

De lo anteriormente señalado, es posible deducir que la regeneración ósea es posible de lograr mediante el uso de biomateriales, los que se han desarrollado gracias a la Ingeniería de Tejidos, que es definida como: la aplicación de los principios y métodos de la ingeniería y ciencias de la vida para la comprensión fundamental de la estructura, función y relaciones en patología y normalidad de los tejidos de los mamíferos, así como para el desarrollo de sustitutos biológicos para restaurar, mantener o mejorar funciones de los organismos (Hutmacher et al., 2007).

Los sustitutos biológicos deben desarrollar una serie de funciones vitales requeridas para desarrollar respuestas dirigidas en el huésped. Estas funciones pueden ser en temas de ingeniería o en temas biológicos (Brekke et al., 1998).

Las funciones en temas de ingeniería se refieren a que los sustitutos deben mantener un sustrato celular vivo, lo que incluye mantención de la totalidad de las propiedades mecánicas, conservación de las características estructurales internas y mantener la geometría tridimensional (Brekke et $a l$.). Las últimas dos permitirán al biomaterial mantener el espacio planificado y/o diseñado por el clínico en forma y tamaño para que el tejido óseo posteriormente ocupe ese lugar.

Las funciones biológicas se refieren a una serie de acontecimientos que incluye: a) migración celular a la zona del biomaterial, b) unión celular (a superficies sintéticas, glicosaminoglicanos y proteínas de la matriz extracelular, c) mantenimiento de una adecuada circulación colateral, d) desarrollo y mantenimiento de un apropiado fenotipo celular, y e) liberación de proteínas morfogenéticas y proteínas reguladoras (Brekke et al.)

Es así como los biomateriales que cumplen con las características antes señaladas y que son utilizados en regeneración ósea, serán clasificados de la siguiente forma (Olate et al.):

1. Autoinjertos (AI): Hueso obtenido del mismo paciente. 2. Aloinjertos (AL): Biomaterial procesado para ser incorporado a sujetos de la misma especie (Freeze - Dried Bone Allograft o FDBA; Demineralized Freeze - Dried Bone Allograft o DFDBA; Proteína morfogenética o BMP).

3. Aloplásticos (AP): Sustitutos óseos sintéticos (Cerámicas, polímeros hidroxiapatita o HA, fosfato tricálcico o TCP, sulfato de calcio).

4. Xenoinjertos (XE o matrices óseas): Biomaterial procesado a partir de especies no humanas (Bio-Oss, Proteína ósea morfogenética bovina o bBMP).

Ante la disponibilidad de esta variada gama de posibilidades, la toma de decisiones en cuanto a la técnica a utilizar, que implica además elegir los biomateriales necesarios en cada una de ellas, influirá en el pronóstico del procedimiento.

En consideración a lo expuesto, el biomaterial con mejores propiedades para lograr regeneración ósea, es el AI; sin embargo, existe una alternativa a éste, sería realizar un AL, los cuales corresponden a preparados óseos procedentes de bancos de tejidos. Este tipo de injerto puede encontrarse en dos formas: una mineralizada, FDBA (Freeze - Dried Bone Allograft) y la variante desmineralizada DFDBA (Demineralized Freeze - Dried Bone Allograft) (Olate et al.; Anitua; Fuentes et al.).

Existe además, la alternativa de utilizar matrices óseas (XE), que se encuentran indicadas en casos donde la cantidad celular y actividad mitótica del lecho receptor es suficiente para poblar la matriz y regenerar el tejido. Ejemplo de este tipo de material es el hueso mineral bovino (Bio - Oss®) (Olate et al.; Anitua; Fuentes et al.).

Se ha señalado que, dentro de los tipos de biomateriales antes citados, los injertos óseos obtenidos del propio paciente serían los que poseen un mejor pronóstico (Anitua et al.; Wong \& Rabie, 1999; Rabie et al., 2000; Kim et al., 2001; Lu et al., 2002; Zoricic et al., 2002; Bidic et al., 2003; Cordioli et al., 2003; Butterfield et al., 2004; Chacón et al., 2004); Stubbs et al., 2004; Donos et al., 2005; Wong et al., 2005; Okazaki et al., 2005), transformándose en el material de referencia (Gold Standard) a la hora de probar biomateriales en regeneración ósea (Olate et al.). De hecho, los AI de origen endocondral (EC) e intramembranoso (IM) presentan, respectivamente, un $357 \%$ y un $642 \%$ más de formación ósea que un AL de tipo IM ( $\mathrm{Lu} \&$ Rabie 2002).

A su vez, los AI de tipo IM son más exitosos en la neoformación ósea que sus homólogos de tipo EC (Lu et al., 2002, Donos et al.; Bidic et al.), Esto se debe a que los primeros mantienen un volumen más estable en el tiempo (Donos et al.), porque presentan una menor reabsorción (Lu et al., 2004, Bidic et al.), de hecho, se ha cuantificado en un $65 \%$ la pérdida de volumen óseo de un AI de origen EC en comparación con el hueso IM (Lu et al. 2004, Wong et al.), asimismo, este último produce $166 \%$ más neoformación ósea que el EC (Wong \& Rabie). Esta situa- 
ción puede explicarse dado que el EC adopta una ruta de osificación que pasa por cartílago en una primera instancia, a diferencia del IM, que osifica directamente (Wong \& Rabie), o bien por la revascularización temprana y extendida que presenta el injerto intramembranoso en comparación al endocondral (Wong \& Rabie).

Si bien es cierto, la evidencia señala que las posibilidades de éxito con AI son altas, existen factores a considerar que sería limitante a su uso: el abastecimiento y disponibilidad son limitados, por lo que, en ocasiones, es necesario utilizar biomateriales como sustitutos óseos (Wong \& Rabie, 1999; Rabbie et al., 2000a,b; Lu et al., 2002; Donos et al.; Lu et al., 2004; Bidic et al.; Zoricic et al.; Butterfield et al.; Kim et al.; Stubbs et al.; Wong et al., 2005; Okazaki et al.; Cordioli et al.; Chacón et al.).

Como fue señalado, se consideran características ideales de biomateriales sustitutos óseos que sean firmes, maleables, osteoconductivos, osteoinductivos, reabsorbibles, inexpansivos y fáciles de usar intraoperatoriamente. Además, deben promover la adhesión, proliferación y diferenciación celular, debe ser también un efectivo transportador y liberador de sustancias y células, así como facilitar el crecimiento y establecer un ambiente que soporte la regeneración ósea (Bidic et al.).

Se ha señalado que la matriz ósea intramembranosa posee altas propiedades osteoinductivas y realza la integración de injertos de hueso intramembranoso autólogo, en conejos (Lu \& Rabie., 2002).

Hueso alogénico mezclado con matriz ósea desmineralizada produce un $224 \%$ más hueso que el hueso alogénico intramembranoso solo, donde la matriz podría aumentar la capacidad de las células de producir hueso, e inducir diferenciación de precursores osteogénicos indiferenciados (Lu et al., 2002).

En relación al hueso alogénico disponible en bancos, Zoricic et al. analizaron este tipo de biomaterial tratado de tres formas diferentes: pasteurizado, autoclavado y congelado. Señalan que la mayoría de las partículas de aloinjertos pasteurizado y congelados se reabsorben e incorporan completamente luego de 4 semanas, mientas que las partículas autoclavadas se encuentran aún intactas y sin reabsorber en este período de tiempo. Del mismo modo, las partículas pasteurizadas son tan rápidas en osteo-inducción como lo son las congeladas.

El hueso neoformado raramente fue hueso laminar luego de 4 semanas. Los huesos pasteurizado y congelado formaron puentes sobre zonas del defecto óseo.
El tejido neoformado creó continuidad con el tejido óseo del huésped, donde la osteoinducción fue pronunciada (Zoricic et al.).

Los nuevos tejidos endosteal, periosteal y perivascular fueron observados en huesos pasteurizado y congelado, con menores diferencias morfológicas a las dos semanas y reacciones más fuertes a las 4 semanas (Zoricic et al.).

El grosor osteoide y número de osteoblastos no variaron considerablemente entre hueso, congelado y pasteurizado, pero fueron significativamente mayores en relación al hueso autoclavado (Zoricic et al.).

La utilización de matrices óseas ha sido también una variable en regeneración ósea. Rabie et al., 2000, han señalado que las matrices óseas desmineralizadas poseen altas propiedades osteoinductivas, y aumenta considerablemente la integración de injertos de hueso autógeno en experiencias realizadas en cráneos de conejos (Rabie et al., 2000a,b). En este marco, el uso de hueso bovino desproteinizado también ha sido satisfactoriamente utilizado en regeneración ósea (Okazaki et al., 2005).

Stubbs et al. han utilizado matrices óseas en experiencias en animales, un sustituto de hueso formado por carbonato de calcio hidroxiapatita poroso solo y en combinación con sulfato de calcio. Se encontró formación de hueso reticular nuevo a las 24 semanas. El sulfato de calcio mejora el manejo del carbonato de calcio hidroxiapatita poroso durante la cirugía y no tiene algún efecto adverso en la formación ósea a corto o largo plazo. Fue soportado el crecimiento interno de hueso nuevo en su porción porosa. La reabsorción de sulfato de calcio fue recién completa a las 6 semanas y podría no ser encontrado antes de este tiempo (Stubbs et al.).

En relación a la utilización de membranas en regeneración ósea, Donos et al. señalan que la estabilidad a largo plazo de un injerto óseo intramembranoso y endocondral combinado con membrana, es superior a la colocación del injerto solo.

En ambos casos, los injertos mantuvieron su volumen inicial y resultaron con un crecimiento predecible, en comparación a los injertos solos (Donos et al.).

A los 5 meses, la membrana fue completamente integrada al nuevo hueso que rellenaba por completo el área creada bajo ésta, donde no era posible distinguir el límite del injerto y el hueso nuevo (Donos et al.). 
El área de hueso que no recibió membrana presentó reabsorciones en todas las dimensiones (Donos et al.).

La membrana formó un espacio apartado, donde los injertos se rodearon de hueso y fueron integrados a éste, formado por células de la mandíbula (Donos et al.).

La estabilidad otorgada por la membrana posee una importancia extrema por la cantidad de hueso neoformado, ya que la movilización de la membrana produce migración de tejido conectivo bajo ésta, y reabsorción del injerto (Donos et al.), por lo que los injertos y membranas podrían ser fijados. Una alternativa podrían ser los pernos reabsorbibles (Chacón et al., 2004).

A los 11 meses post implantación, ambos tipos de hueso tratado, con membrana exhibieron disminución de sus dimensiones comparados con lo observado a los 5 meses. Eso es esperable, ya que se ha señalado que al remover la membrana ocurre reabsorción, la que va acompañada de maduración ósea y formación de mayor trabeculado. El trauma de la segunda cirugía para la remoción de la membrana puede ser responsable de la reabsorción ósea (Donos et al.).

La membrana previene la reabsorción del injerto independiente del origen embriológico de él, lo que señala que lo más importante para mantener un injerto es asegurar que éste sea colonizado por células osteogénicas.

Cubrir los injertos óseos intramembranosos y endocondrales mejora la predictibilidad y la estabilidad a largo plazo de los injertos, probablemente al asegurar la colonización de los injertos con células osteogénicas del sitio receptor (Donos et al.; Butterfield et al.).

En relación al uso de membrana Okazaki et al. señalaron que la presencia de coágulos sanguíneos en los espacios del injerto promueve la neoformación ósea en estudios in vivo y clínicos. Del mismo modo, el tejido osteoide neoformado cambia rápidamente por hueso laminar a 8 semanas en estudios experimentales. Ha sido confirmada la rápida neoformación ósea al principio del tratamiento, así como se evidencia la presencia de diferentes patrones de neoformación ósea en la región bajo la membrana (Butterfield et al.).

Fueron definidos por estos autores los límites del crecimiento vertical del hueso injertado, que fue de 1,5 a $5 \mathrm{~mm} ; 1,5 \mathrm{~mm}$ era equivalente al $30 \%$ del crecimiento total, lo que resulta de un insuficiente tiempo de curación. Se desconoce el tiempo exacto requerido por los proce- dimientos que utilizan membrana para la cicatrización ósea total (Butterfield et al.).

\section{DISCUSIÓN}

En la literatura consultada es posible obtener valiosa información con respecto a la utilización, pronóstico y resultado de terapias de regeneración ósea con diversos tipos de biomatetiales, sean estos autoinjertos, aloinjertos, xenoinjertos o materiales aloplásticos solos (no asociados con otros biomateriales), así como combinados entre ellos y asociados al uso de matrices y/o membranas, situación de utilidad en cuanto a orientar en la toma de decisiones en el tipo de material y técnica a usar para la reconstrucción del reborde maxilar exiguo. No obstante, la literatura consultada no muestra investigaciones que evalúen varios tipos de biomateriales con y sin la utilización de membranas, en un grupo de individuos.

La posibilidad de establecer el éxito de una terapia de regeneración ósea puede ser evaluada histológicamente; sin embargo, las publicaciones analizadas no entregan herramientas de diagnóstico menos invasivas para el paciente que orienten al clínico en cuanto al éxito relativo de los procedimientos realizados.

Sería interesante desarrollar investigaciones en esta área, que busquen establecer las potenciales relaciones entre la evolución histológica y la imagen radiográfica (densidad de la imagen) en el uso de biomateriales en regeneración ósea, considerando la necesidad de evaluar clínicamente el resultado del tratamiento de regeneración del hueso previo a la realización de procedimientos de rehabilitación oral implanto asistida, sin dejar de lado la documentada presencia de errores entre lo que este examen muestra y los hallazgos en la cirugía (Álvarez Cantoni et al., 2008).

Los AI han reportado ser los biomateriales más exitosos a ser usados en terapias de regeneración ósea. Dada su baja disponibilidad y la necesidad de poseer entrenamiento clínico en la cosecha de él, pueden ser usados en conjunto con matrices óseas o xenoinjertos. Las membranas han mostrado aumentar la estabilidad de los biomateriales, con un posible potencial mejor pronóstico en este tipo de terapias.

Los antecedentes antes presentados pueden ser una valiosa herramienta de orientación a los profesionales del área de la cirugía maxilofacial y rehabilitación oral, en cuanto a la toma de decisiones clínicas en terapias de regeneración ósea maxilo - mandibular. 
OPORTO, V. G.; FUENTES, F. R.; ÁLVAREZ, C. H. \& BORIE, E. E. Maxillomandibular morphology and physiology recovery: Biomaterials in bone regeneration. Int. J. Morphol., 26(4): 853-859, 2008.

SUMMARY: Dental caries and periodontal disease are public heath problems. 100\% of people from low-medium and low socio economic levels have caries. $92.19 \%$ of people between 35 and 74 years of age have periodontal disease. Both pathologies may cause teeth fall down. Titanium implants could improve prognosis of prosthetics treatments, but frequently requires increase bone volume, to restore shape and function. The research wants to know state of art in use of biomaterials in bone regeneration. Best biomaterial properties were shown by autologous bone $(\mathrm{AB})$, however, do exist alternatives such as allograft (AL) and bone matrix (BM). AB has shown best prognosis, from endochondral (EC) and intramembranous (IM), with 357\% and 642\% most bone formation compared to AL IM. AB IM type is successfully compared to EC (IM shown $166 \%$ more bone formation). AB access is limited, this fact have made necessary use other biomaterials. AL combined with BM produce $224 \%$ more bone compared to AL. Membrane use improves long term stability of IM and EC bone. AB EC type is the most successful biomaterial. Considering limited access to this bone, it could be used combined with bone matrix. Membranes have shown increase long term stability of biomaterials.

KEYWORDS: Bone regeneration; Biocompatible materials; Dental implants.

\section{REFERENCIAS BIBLIOGRÁFICAS}

Álvarez Cantoni et al. Estudio retrospectivo de la anatomía topográfica y de hallazgos en ambos maxilares realizados con tomografía axial computada (T.A.C. 3D). Dental Dialogue, 1: 46-61, 2008.

Anitua, E. \& Andia, I. Un nuevo enfoque en la regeneración ósea plasma rico en factores de crecimiento $(P$. R. G. F). Editorial Puesta al Día Publicaciones, S.L, 49-78, 2000.

Bidic, S. M. S.; Calvert, J.; Marra, K.; Kumta, P.; Campbell, P.; Mitchell, R.; Wigginton, W.; Hollinger, J.; Weiss, L. \& Mooney, M. Rabbit calvial wound healing by means of seeded caprotite scaffolds". J. Dent. Res., 82 (2):131-5, 2003.

Brekke J, Toth J. Principles of Tissue Engineering Applied to Programmable Osteogenesis. J. Biomed. Mater. Res. (Appl Biomater), 43:380-98, 1998.

Butterfield, K.; Bennett, J.; Gronowicz, G. \& Adams, D. Effect of PRP with autogenous bone graft for maxillary sinus augmentation in a rabbit model. J. Oral Maxillofac. Surg., 63:370-6, 2004.

Cordioli, G.; Atiyeh, F.; Piatelli, A. \& Majzoub, Z. Healing of transplanted composite bone grafts-implants. Clin. Oral Impl. Res., 14:75-8, 2003.

Chacón, G.; Ellis, J.; Kalamar, J. \& Mc Glumphy, E. Using resorbable screws for fixation of cortical onlay bone grafts: An in vivo study in rabbits. J. Oral Maxillofac., Surg. 62:1396-402, 2004.
Donos, N.; Kostopoulos, L.; Tonetti, M. \& Karring, T. Long term stability of autogenous bone grafts following combined application with guided bone regeneration. Clin. Oral. Impl. Res., 16:133-9, 2005.

Fuentes, R.; Weber, B. \& Soto M. Revisión bibliográfica de rellenos de seno maxilar previo a implantes oseointegrados. A propósito de un Caso Clínico. Rev. Chil. Cs. Med. Biol. 15(2):63-6, 2005.

Gamonal, J, A.; López, N. J. \& Aranda, W. Periodontal conditions and treatment needs, by CPITN, in the 35-44 and 65-74 year-old population in Santiago, Chile. Int. Dent. J., 48: 96-103, 1998.

Hutmacher, D.; Schantz, J.; Xu Fu Lam Ch; Cheng Tan, K. $\&$ Chye Lim T. State of the art and future directions of scaffold-based bone engineering from a biomaterials perspective. J. Tissue Eng. Regen. Med., 1: 245-60, 2007.

Kim, E.; Park, E. \& Choung, P. Platelet concentration and its effect on bone formation in calvarial defects: An experimental study in rabbits. J. Prosthet. Dent., 86:428 $33,2001$.

Lu, M. \& Rabie, B. The effect of demineralized intramembranous bone matrix and basic fibroblast growth factor on the healing of allogenic intramembranous bone graft in the rabbit. Archives of Oral Biology, 47:831-41. 2002.

Lu, M. \& Rabbie, A. B. M. Quantitative assessment of early healing of early healing of intramembranous and 
OPORTO, V. G.; FUENTES, F. R.; ÁLVAREZ, C. H. \& BORIE, E. E. Recuperación de la morfología y fisiología maxilomandibular: Biomateriales en regeneración ósea. Int. J. Morphol., 26(4): 853-859, 2008

endochondral autogenous bone grafts using microcomputed tomography and Q- Win image analyzer. Int. J. Oral Maxillofac. Surg., 33:369, 376. 2002.

MINSAL. Encuesta Nacional de Salud 2000. Santiago. Marzo 2004.

Okazaki, K.; Shimizu, Y.; Xu, H. \& Ooya, K. Blood-Filled spaces with and without deproteinized bone grafts in guided bone regeneration. Clin. Oral Impl. Res., 16:236 $-43,2005$.

Olate, S.; De Olivera, G.; Jaimes, M. \& Barbosa, J. Reparación ósea en procedimientos de reconstrucción y colocación de implantes. Int. J. Morphol., 25(3):649-57, 2007.

Rabbie, A. B. M.; Wong, W. \& Hägg, U. Composite autogenous bone and demineralized bone matrices used to repair defects in the parietal bone of rabbits. British J. of Oral and Maxillofacial Surgery, 38:565 -70, 2000.

Rabie, A.; Han Chay, S. \& Wong, A. Healing of autogenous intramembranous bone in presence and abscence of homologus demineralized intramembranous bone. Am. J. Orthod Dentofacial Orthop., 117: 288 -97, 2000.

Stubbs, D.; Deakin, M.; Champan-Sheath, P.; Bruce, W.; Debes, J.; Gillies, R. \& Walsh, W. In Vivo evaluation of resorbable bone graft substitutes in a rabbit tibial defect model. Biomaterials, 25:5037-44, 2004.

Urbina, T.; Caro, J. P. \& Vicent, M. Caries y fluorosis en niños de 6 a 8 años y 12 años. Minsal, Chile. Departamento de Estadísticas e Información. 1996-1999.

Villagrán, E.; Linossier, A. \& Donoso, E. Count of salivary Streptococci mutans in pregnant women of the metropolitan region of Chile: cross-sectional study. Rev. Méd. Chile, 127:165-70, 1999.

Wong, R. \& Rabie, B. A quantitative assessment of healing of intramembranous and endochondral autogenous bone grafts. European J. of Orthodontics 21:119-26, 1999.

Wong, R. \& Rabie, B. Histologic and ultrastructural study on statin graft in rabbit skulls. J. Oral Maxillofac. Surg., 63:1515-21, 2005.

Zoricic, S.; Bobinac, D.; Lah, B.; Maric, I.; Cvijanovic, O.; Bajec, S.; Golubovic, V. \& Mihelic, R. Study of the healing process after transplantation of pasteurized bone graft in rabbits. Acta Med. Okayama 56, (3):121-8, 2002.
Dirección para correspondencia:

Gonzalo Oporto Venegas

Cirujano Dentista

Facultad de Medicina Universidad de La Frontera

Manuel Montt 112

Temuco - CHILE

Email: goporto@ufro.cl

Recibido : 16-09-2008

Aceptado: $20-10-2008$ 
